\title{
A comprehensive assessment of breast and cervical cancer control infrastructure in Zambia
}

\author{
Carla Chibwesha ${ }^{a}$, Leeya F. Pinder ${ }^{a, b}$, Agnes Musonda ${ }^{c}$, Kombatende Sikombe ${ }^{c}$, Jane Matambo ${ }^{c}$, \\ Allen C. Bateman ${ }^{\mathrm{a}}$, Claire-Helene Mershon ${ }^{\mathrm{c}}$, Mulindi Mwanahamuntu ${ }^{\mathrm{b}}$, Sharon Kapambwe ${ }^{\mathrm{c}}$, \\ Kennedy Lishimpi ${ }^{\mathrm{d}}$, Groesbeck P. Parham ${ }^{\mathrm{a}, \mathrm{b}, *}$ \\ a University of North Carolina at Chapel Hill, Department of Obstetrics and Gynecology, 101 Manning Drive Chapel Hill, NC 27514 USA \\ b University Teaching Hospital, Department of Obstetrics and Gynaecology, Nationalist Road, P/Bag RW1X, Lusaka 10101, Zambia \\ c Center for Infectious Diseases Research in Zambia, Box 34681, Lusaka 10101 Zambia \\ d Cancer Diseases Hospital, P.O. Box 51337, Lusaka 10101 Zambia
}

\section{A R T I C L E I N F O}

\section{Keywords:}

Global health

Cervical cancer

Breast cancer

Healthcare quality

Access

Evaluation

Healthcare utilization

\begin{abstract}
A B S T R A C T
Introduction: By 2030 cancer will kill one million Africans each year. Women will bear the heaviest burden, as cancers of the breast and cervix are the most common malignancies and causes of cancer-related death in the African region. National-level data that map the status of women's cancer control services are needed to inform strategies for implementing platforms for the early detection and treatment of these "priority" cancers.

Methods: Using mixed-methods, we assessed available services for breast and cervical cancer detection and treatment at all provincial hospitals, the national referral hospital, and the national cancer treatment center in Zambia.

Results: A system for cervical cancer prevention using visual inspection with acetic acid (VIA) and ablation/ excision of precancerous lesions has been established at the provincial level. The potential for mammography, clinical breast examination, diagnostic ultrasound and biopsy exist at the provincial level, albeit on a much smaller scale. Breast wedge resections and mastectomy can be performed in provinces where general surgeons are located; however, breast conserving and reconstructive surgery are not available. Invasive cancers are generally referred to University Teaching Hospital in Lusaka, where services for radiation, chemotherapy and hormonal therapy are available but overburdened. Pathology services nationwide are woefully inadequate.

Discussion: The assessment revealed a critical need for centrally coordinated, but decentralized, public service platforms for women's cancer control. Efforts are underway, through multiple stakeholders, to implement recommendations related to training healthcare workers who can provide advanced diagnostic and therapeutic services, improving pathology services, and innovative financing for these initiatives.
\end{abstract}

\section{Introduction}

The growing global cancer burden is increasingly falling on lowand middle-income countries (LMICs), where nearly $80 \%$ of disability adjusted life-years-lost to cancer occurs [1-4]. In Africa the need for action is profound as it is predicted that by 2030 cancer will kill one million Africans each year [2]. Women will bear the heaviest burden, as cancers of the breast and cervix are the most common malignancies and causes of cancer-related death in the African region.

\section{Background}

Breast cancer incidence rates in developing nations, although rising, are much lower than those in developed nations. Mortality rates, however, are just as high. System level barriers to breast cancer control in LMICs are centered around the lack of screening, early detection, diagnostic, and treatment facilities [5]. Although scarce, Zambia-specific data indicates that breast cancer incidence has been rising.

Cervical cancer is a major cause of cancer deaths among women in LMICs. One in five cases reported globally occurs in sub-Saharan Africa $[6,7]$. System level barriers are similar to those for breast cancer. HIV infected women are at increased risk of developing cervical precancer and cancer. [8-10]. Zambia has disproportionately high cervical cancer incidence and mortality rates $[4,11]$, as well as a generalized HIV/AIDS epidemic [12].

\footnotetext{
* Corresponding author at: University Teaching Hospital, Department of Obstetrics and Gynaecology, Nationalist Road, P/Bag RW1X, Lusaka 10101, Zambia.

E-mail address: professorparham@gmail.com (G.P. Parham).
} 


\section{Preliminary activities}

In 2005 the Zambian Ministry of Health facilitated the initiation of the Cervical Cancer Prevention Program in Zambia (CCPPZ). Since inception the CCPPZ has implemented nurse-led public cervical cancer prevention service platforms where screening is performed by visual inspection with acetic acid (VIA), followed by ablation with cryotherapy or thermal coagulation, excision with loop electrical excision procedure (LEEP) or referral for biopsy [13]. To date CCPPZ has screened over 500,000 women [14-16]. Supported by the U.S. President's Emergency Plan for AIDS Relief (PEPFAR) through the Centers for Disease Control and Prevention (CDC), as well as a prominent public-private partnership - Pink Ribbon Red Ribbon - the CCPPZ was able to build a highly efficient management and quality assurance infrastructure. [14-16]

More recently, the Zambian government has accelerated its efforts to expand breast cancer early detection and treatment capacity through the initiation of a partner-sponsored effort with the Susan G. Komen Breast Cancer Foundation. The model consists of breast cancer public awareness campaigns, clinical breast examination (CBE) by nurses, ultrasound-guided biopsy of palpable breast masses by high-level healthcare providers and referral for treatment. However, limited funding and the lack of an efficient delivery and management system preclude the availability of these services, except on a very small scale.

Despite these initiatives, there are no national-level data that map women's cancer control services in the country.

\section{Methods}

The overall aim of this project was to quantify existing service delivery capacity and to identify gaps, challenges, and priority areas of focus for building setting-appropriate and sustainable breast and cervical cancer control service platforms throughout Zambia.

The project provides an overview of services currently available for breast and cervical cancer screening, early detection, diagnosis and treatment in Zambia, obtained through a nationwide survey. The survey was accomplished by conducting health facility assessments (HFAs) at all 9 provincial hospitals in Zambia, as well as at the University Teaching Hospital (UTH) and Cancer Diseases Hospital (CDH) in the Lusaka Province. Provincial and tertiary facilities were the focus of the assessment because they have been identified by the Zambian National Cancer Control Strategic Plan as the highest priority facilities for expansion of cancer screening, early detection, and treatment services.

For each facility surveyed we assessed human resources (health care providers with specialized training in breast and cervical cancer screening, early detection and treatment) and facility infrastructure (capacity, equipment, and supplies) to provide breast and cervical cancer screening, early detection and treatment services, as well as HPV vaccination services. Relevant referral systems were also assessed. Concurrent with facility assessments, we conducted a structured interview with each Provincial Health Officer, which included questions on service provision, utilization, and costs to a patient for receiving breast or cervical cancer screening, early detection, diagnosis, and treatment in each district of the particular province. The availability of supplies and equipment was also evaluated at the district level but was not substantiated through visual inspection. All fieldwork was completed between August 2014 and January 2015.

\section{Results}

\subsection{Population}

Zambia is divided into 10 provinces, comprised of 102 districts, with a total population of $14,891,010$. The target population for the assessment was women aged $25-59$ years ( $\mathrm{n}=2,208,870$ ), who comprise $\sim 15 \%$ of Zambia's population. Provincial Health Offices reported
1937 health facilities within the country, corresponding to a national rate of 1.3 health facilities per 10,000. Although there are no established benchmarks for the ideal number of health facilities, hospitals, or hospital beds per 10,000 population, the World Health Organization reports an average of 0.8 hospitals per 10,000 for the African region [17].

\subsection{Human resources}

The paucity of human resources for health has previously been noted as a limitation in patients' access to breast and cervical cancer care in Zambia. The Provincial Health Offices reported a total of 11,746 healthcare workers, including nurses $(n=9609)$, clinical officers $(n=1179)$, general medical officers $(n=665)$, specialists $(n=104)$, technologists $(n=109)$, and pharmacists or pharmacy technicians ( $n=80$ ). Of these healthcare workers, $2955(26 \%)$ are employed in the provincial, tertiary, and specialty hospitals (2587 nurses, 249 general medical officers, and 119 clinical officers). The provincial and tertiary hospitals surveyed reported an additional 104 specialists (40 general surgeons, 28 gynecologists, 6 clinical oncologists, 14 anesthesiologists, 7 radiologists, 9 pathologists), and 109 technologists (36 anesthetists, 42 radiographers, 27 histology technologists, and 4 cytology technicians) (Fig. 1). The largest cadre of healthcare workers in the public sector is nurses, who account for $82 \%$ of the total healthcare workers, while specialists and technologists make up only $2 \%$.

\subsection{Service distribution}

In most provinces, the provincial hospital is located in the provincial capital and also serves as the district hospital for that district. In this analysis, all services provided at the provincial hospital are counted toward services provided in that district.

Breast cancer screening (mammography), early detection (CBE) and diagnostic (ultrasound, biopsy, pathology) services are not universally available. Six out of 10 provinces have the potential capacity to offer CBE and either mammography or ultrasound; neither Muchinga nor Luapula Provinces report offering of any of these services. (Fig. 2).

All provincial hospitals provide cervical cancer "screen and treat" services or refer to a nearby clinic; as a result, all provinces have at least one district providing cervical cancer prevention. All provinces also have at least one district outside the capital that provides cervical cancer prevention services, with the exception of Muchinga Province. Fig. 5 shows the provinces providing cervical cancer prevention in at least one facility.

\subsection{Breast cancer services}

With respect to early detection and screening services, healthcare workers trained to perform either $\mathrm{CBE}$, ultrasound or mammography are present in 8 of 11 hospitals. Two of the hospitals surveyed do not have the capacity for CBE, ultrasound, or mammography (Fig. 2).

Three hospitals have dedicated breast specialty clinics, each of which offers CBE, mammography, ultrasound and ultrasound-guided breast biopsy (fine needle aspiration and core needle biopsy). These clinics operate in Kabwe General Hospital (Central Province) one day per week, University Teaching Hospital (Lusaka Province) every weekday, and Cancer Diseases Hospital (Lusaka Province) three days per week. Only 5 of the 11 hospitals were able to report numbers of women assessed for breast cancer in the 12-month period preceding the survey. The total number reported was 1414; of these, 839 ( $\sim 60 \%)$ were screened at the Cancer Diseases Hospital in Lusaka.

The most common forms of breast cancer evaluation and treatment are incisional or excisional wedge biopsy and mastectomy, both of which are performed at 8 hospitals. One hospital charges for these services. Radiation therapy is offered only at the Cancer Diseases Hospital. Chemotherapy is offered at 4 hospitals, but mainly provided 


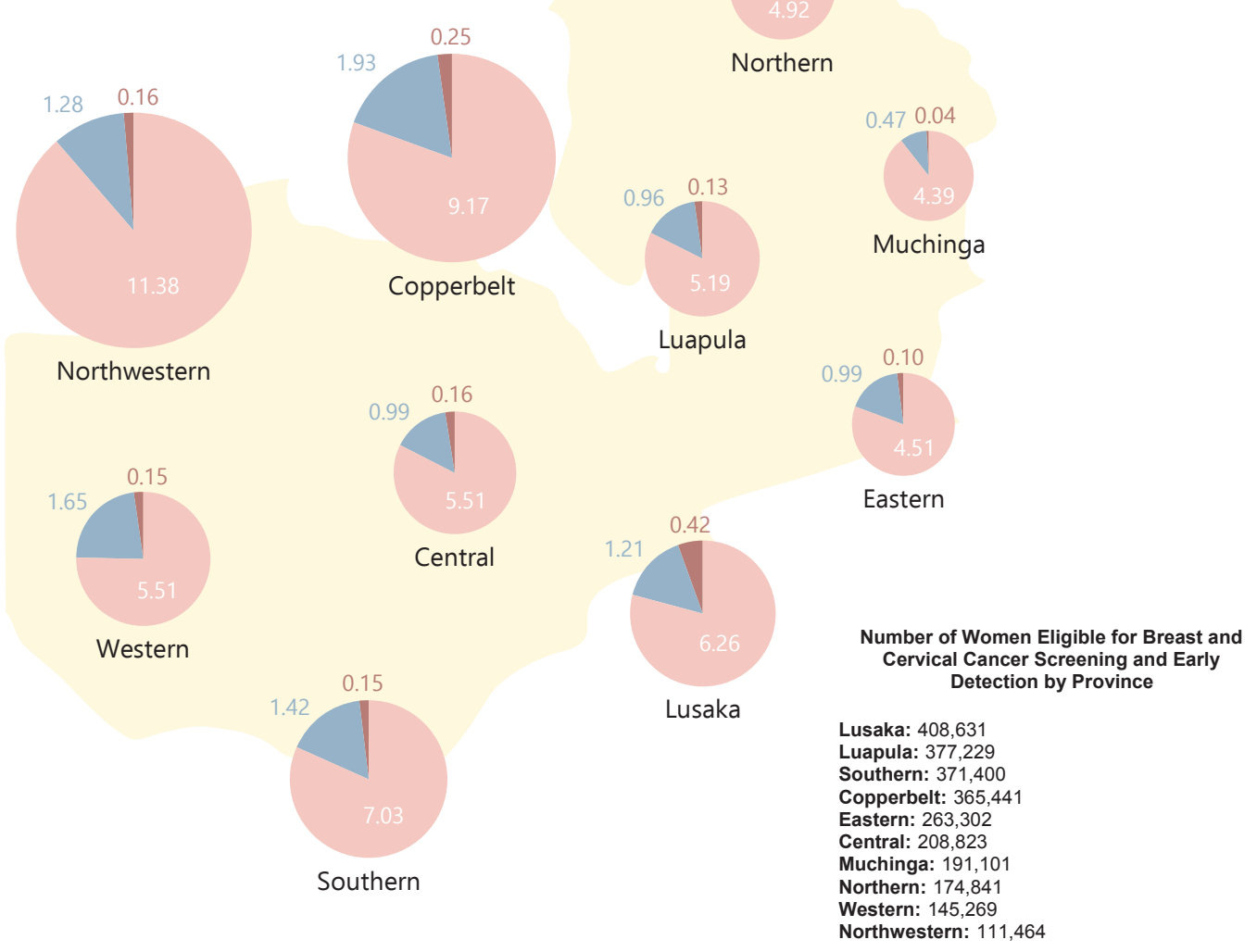

at the Cancer Diseases Hospital. Endocrine (hormonal) therapy is offered only at the Cancer Diseases Hospital.

Only a small proportion of the frontline healthcare workforce has been trained to provide breast cancer specialty services. Of 2587 nurses and midwives in the hospitals surveyed, 628 (24\%) are trained in CBE, but only 137 (5\%) provide it. Of 249 General Medical Officers, 104 (42\%) are trained in CBE and 91 (37\%) provide it. The majority of gynecologists are trained in (26; 93\%) and reportedly provide CBE (27; $96 \%)$. Among 40 general surgeons, $22(55 \%)$ are trained in incisional or excisional wedge biopsy and mastectomy.

In this assessment, 9 of the hospitals reported having at least 1 mammography machine (10 machines in total, with 6 hospitals with analog machines, 2 with digital machines, and 1 with both an analog and a digital machine). Of the 9 hospitals with mammography machines, 7 machines were functional. All facilities reported at least one functioning ultrasound machine (Fig. 3). However, we did not verify
Fig. 1. Number of Healthcare Personnel Employed per 10,000 Population by Province. the functional status of mammography and ultrasound machines, nor whether ultrasound machines were equipped with the special probes required for scanning the breasts.

There were no major gaps reported in availability of surgical equipment or supplies for surgery or incisional/excisional breast biopsy. All surgical facilities reported available and functional anesthesia and IV equipment, laryngoscopes, oropharyngeal airways, endotracheal tubes, oxygen cylinders, operating tables, anesthetic facemasks, and suction aspirators. All facilities also reported IV solution, scalpels, sutures, and disinfectant currently in stock. CDH reported having radiotherapy equipment. Once again, we did not verify the functional status, quality, or quantity of supplies or equipment.

\subsection{Cervical cancer services}

All 11facilities provide cervical cancer screening, diagnosis, or

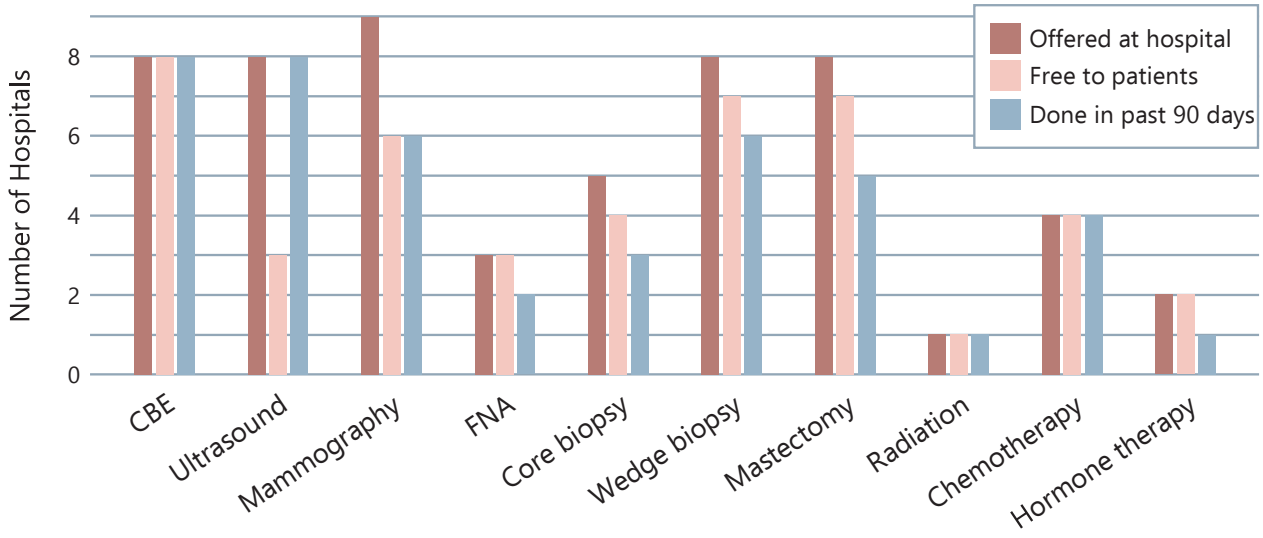

Fig. 2. Breast Cancer Screening, Diagnosis, and Treatment Services at 11 Provincial, Tertiary, and Specialty Hospitals. 


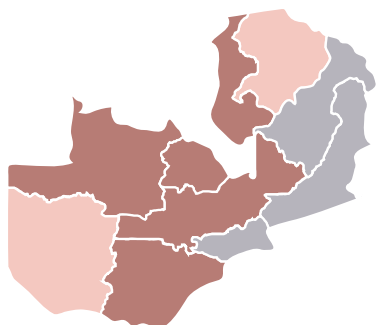

Analog Mammogram

Available and functional

Available but non-functional

Not available

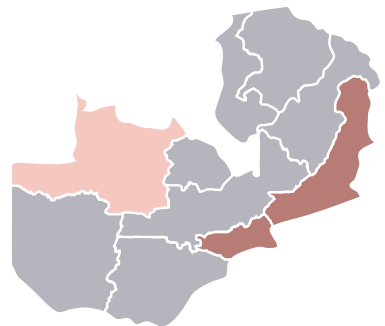

Digital Mammogram

Available and functional

Available but non-functional

Not available

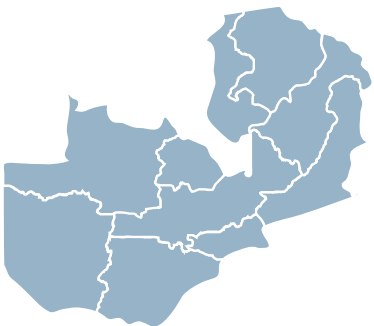

Fig. 3. Availability and Functionality of Mammography and Ultrasonography by Province. treatment of cancer precursors. In the 12-month period preceding the survey, 27,369 women were screened for cervical cancer across the 11 facilities surveyed. An additional 18,432 women were screened at CCPPZ-supported facilities not included in this survey (45,801 in total). While Kabwe General Hospital in the Central Province does not provide cervical cancer screening, it refers patients to a nearby primary health center (Ngungu Clinic), which has an active "screen and treat" program. Two facilities offer cytology-based cervical cancer screening. Nine facilities offer VIA, which is free of charge to patients in all and is conducted by nurses and midwives. None currently offer HPV-based screening. Cervical punch biopsy is offered in 10facilities. For cervical cancer precursors, there are three types of treatment available: 9 of the facilities surveyed provide cryotherapy, 10 provide loop electrical excision procedure (LEEP), and 1 offers cold-knife cervical conization. For cases of early invasive cervical cancer, simple hysterectomy is available at 8 facilities and radical hysterectomy with pelvic lymphadenectomy is available at UTH. As with breast cancer treatment, radiation is available only at $\mathrm{CDH}$. Chemotherapy for cervical cancer treatment is offered at $\mathrm{UTH}, \mathrm{CDH}$, and Livingstone General Hospital, but primarily administered at $\mathrm{CDH}$. Brachytherapy is available only at $\mathrm{CDH}$. Chemoradiation and brachytherapy services are offered free of charge to patients (Fig. 4).

HPV vaccination is not currently offered at any of these hospitals, but was available in four districts in Lusaka Province through an externally funded demonstration project.

Cervical cancer prevention and treatment capacity is distributed among the healthcare workforce in the following manner. Among the 2587 nurses and midwives, $11(<1 \%)$ are trained to perform Pap smears and $31(1 \%)$ VIA, $27(1 \%)$ cryotherapy, and $10(<1 \%)$ LEEP. Only $4(<1 \%)$ nurses are trained in HPV testing. Of 249 General Medical Officers, $20(8 \%)$ are trained to perform Pap smears and 18 (7\%) VIA, 15 (6\%) cryotherapy, 28 (11\%) LEEP, and 45 (18\%) cervical punch biopsies. Almost all of the gynecologists are trained to perform Pap smears and cold-knife conization, 21 (75\%) VIA, and 20 (71\%)

\section{Ultrasound Machine}

Available and functional

Available but non-functional

Not available or no data cryotherapy and LEEP. Of 40 general surgeons, 22 (55\%) are trained in simple hysterectomy. In some facilities, hysterectomies are conducted by visiting gynecologists or expatriate gynecological surgeons, who are not counted in the number of staff at that facility. Overall, only $33(1 \%)$ nurses and midwives and 17 (7\%) GMOs are involved in the provision of cervical cancer prevention services, while 25 (89\%) gynecologists are involved in such.

In this assessment, 9 of the hospitals reported having at least 1 cryotherapy machine and 10 reported having at least 1 LEEP machine. Eight hospitals had at least one functioning digital camera for VIA. (One additional hospital had a non-functioning camera.) Nine hospitals had lithotomy beds, and all hospitals reported having at least 1 speculum. All facilities that perform VIA screening reported having $5 \%$ acetic acid for VIA in stock (Fig. 5).

\subsection{Pathology services}

Three hospitals reported having pathology labs at their facility: UTH, Ndola Central Hospital, and Kabwe General Hospital. Patients pay for pathology services at these facilities. Hospitals without pathology labs send their specimens to private facilities $(n=6)$ and/or UTH $(\mathrm{n}=7$ ). Two hospitals usually receive results within $1-2$ weeks, 6 receive results within 3 or more weeks, and 3 were unable to estimate how long it usually takes to receive results. In the 12-month period preceding the survey, the UTH Pathology Lab processed 1800 specimens, Ndola Central Hospital Pathology Lab processed 2325 specimens, and the Kabwe General Hospital Pathology Lab processed 660 specimens. Key pathology supplies such as $10 \%$ buffered formalin, $\mathrm{H} \& \mathrm{E}$ and Geimsa stain solutions, pipettes, and microtome blades were available. All three facilities' centrifuges, microtomes, and tissue processors were reported as functional.

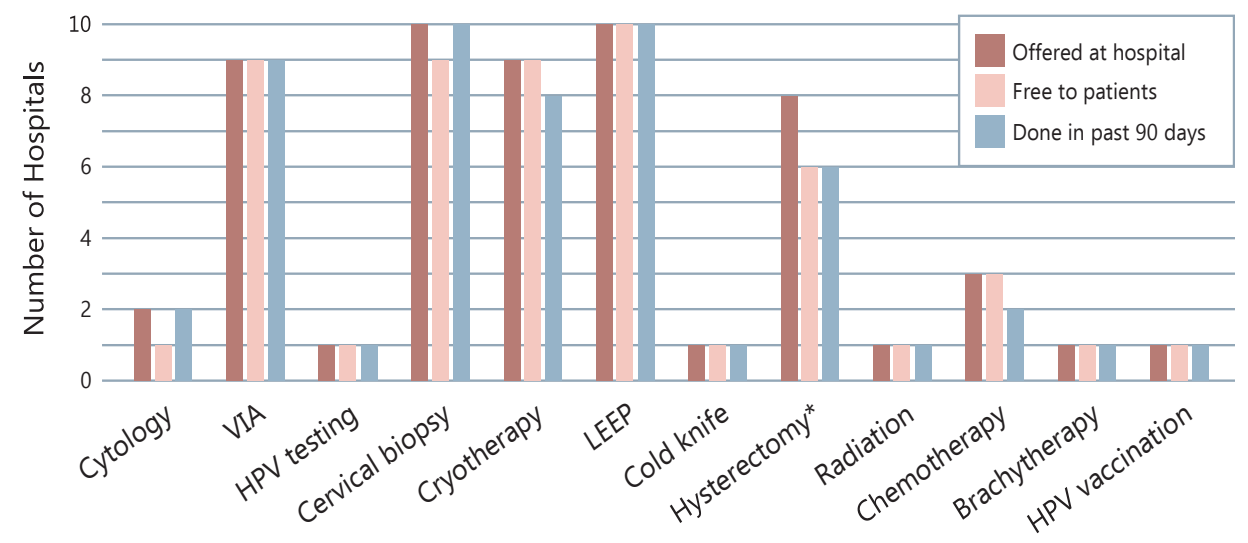

Fig. 4. Cervical Cancer Screening, Diagnosis, and Treatment Services at 11 Provincial, Tertiary, and Specialty Hospitals. 

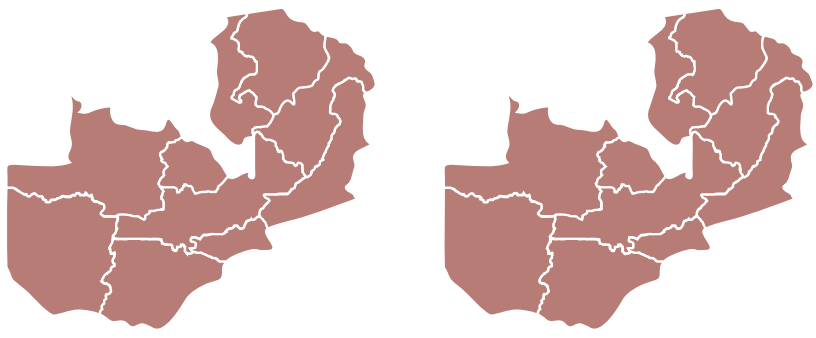

Cryotherapy Machine

Available and functional

Available but non-functional

Not available

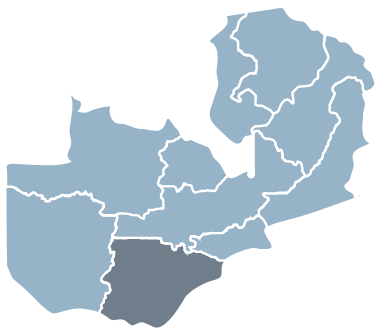

Fig. 5. Availability and Functionality of Cameras for VIA, Cryotherapy Machines, and LEEP Machines by Province.

\subsection{Summary of findings and recommendations}

This report highlights the important cervical cancer prevention gains that have been made in Zambia since the establishment of the CCPPZ in 2005. Notably, cervical cancer screening services are provided in all 10 provinces of Zambia, with over 45,000 Zambian women receiving screening services in the 12-month period preceding our survey. By contrast, breast cancer screening and early detection services are available in 8 of Zambia's 10 provinces, but only 1414 women accessed them in the year preceding the survey. These numbers include women attending both fixed and mobile clinics.

Several challenges common to both cervical and breast cancer services emerged: (i) utilization of screening and early detection services remains low, particularly for breast cancer; (ii) the capacity of provincial- and tertiary-level facilities to further expand and improve the quality of women's cancer services is severely curtailed by a lack of appropriately trained mid- and high-level health personnel, limited funding and pathology services; (iii) existing breast cancer screening and early detection services are not well coordinated; and (iv) advanced therapies (chemotherapy, radiation and oncologic surgery) are concentrated within the Lusaka Province.

Based on the above findings, the survey team provided the following recommendations, many of which have since been implemented, at least partially:

1. Administrative

(1)Establishing an Office of Women's Cancer Control Services responsible for the expansion and coordination of all aspects of women's cancer screening, early detection and diagnostic services in the nation. The office should be supported by the following:

a. National Coordinator - responsible for overall leadership, planning, and administration of the activities of the office

b. Clinical Coordinator - responsible for the coordination and management of all training and clinical activities

c. Provincial Women's Cancer Control Specialists (one per province) responsible for the coordination, implementation, monitoring and evaluation of women's cancer services at the provincial level

These recommendations were accepted, further refined, and implemented by the Zambian Ministry of Health. A new Office of National Cancer Prevention, headquartered at the Ministry of Health, was inaugurated in 2015 and Dr. Sharon Kapambwe, former staff head of CCPPZ, was selected to be the National Cancer Prevention Coordinator. In parallel, the reorganization of UTH has resulted in a separate hospital specifically focused on women's clinical care, directed by the founding co-Director of CCPPZ, Dr. Mulindi Mwanahamuntu. In addition, Dr. Aaron Shibemba, consultant pathologist for CCPPZ, was selected to be the National Coordinator of Pathology and Laboratory services, a new position established in the Ministry of Health, spurred by these recommendations.

11. Programmatic

Immediate

\section{Camera for VIA Imaging}

Available and functional

Available but non-functional

Not available or no data

(1) Create cadres of Women's Cancer Control Trainer of Trainers (TOT) in each province, who will disseminate the following skills to district level health facilities

- Women's cancer health promotion

- Ablative and excisional treatment for cervical precancer

- Clinical breast examination and diagnostic breast ultrasound

- Ultrasound-guided breast biopsy (core needle biopsy and fine needle aspiration)

- Point of care touch preparation cytology of breast biopsy specimens

(2) Convene a Women's Cancer Control Consultative Meeting to determine (a) how best to transition the cervical cancer prevention program from opportunistic to population-based, and (b) the most appropriate model for breast cancer control. The government is presently collaborating with investigators from the University of North Carolina and Research Triangle International to develop models for populationbased cervical cancer screening. The Susan G. Komen Breast Cancer Foundation, in partnership with the Ministry of Health, is sponsoring the consultative meeting in 2017.

(3)Convene a Pathology Consultative Meeting to assess existing pathology and laboratory capacity in the country and develop models for expansion.

(4) Apply for GAVI-supported HPV vaccines. The Ministry of Health has reconvened its efforts to apply for GAVI-supported country-wide HPV vaccination.

\section{Medium Term}

Establish a Women's Oncology Surgical Training Institute at UTH to train surgical oncologists. In July 2015, the Department of Obstetrics and Gynecology at UTH established a Gynecologic Oncology Unit to improve the care of women with gynecologic malignancies. In 2017 the Cancer Diseases Hospital employed a gynecologist and general surgeon, both of whom will undergo specialized surgical oncology training at an accredited cancer care institution in the southern hemisphere in 2017.

Long Term

(1) Expand the graduate pathology training program. The residency program in Pathology at UTH was established in 2011 and the first group of 4 pathology residents completed their training in February 2017. Program expansion efforts are underway.

(2) Expand and strengthen the present national cancer registry to monitor trends in cancer incidence, as well as project future funding and service delivery needs

The Zambian Ministry of Health, as well as stakeholders from the academic, non-profit, and industry sectors, have expressed strong commitments in implementing these recommendations in the short through long-term time horizons. The rapid translation of some of these recommendations as discussed above demonstrate the willpower that exists at all levels to reduce the burden due to these preventable malignancies in Zambia. 


\section{Funding}

This work was supported by the Susan G. Komen Breast Cancer Foundation. Leeya F. Pinder is supported through the UJMT Fogarty Global Health Fellowship, R25TW009340.

\section{References}

[1] F. Bray, et al., Global estimates of cancer prevalence for 27 sites in the adult population in 2008, Int. J. Cancer 132 (5) (2013) 1133-1145.

[2] B.S. Sylla, C.P. Wild, A million africans a year dying from cancer by 2030: what can cancer research and control offer to the continent? Int. J. Cancer 130 (2) (2012) 245-250.

[3] A. Jemal, et al., Global cancer statistics, CA. Cancer J. Clin. 61 (2) (2011) 69-90.

[4] J. Ferlay, et al., Estimates of worldwide burden of cancer in 2008: GLOBOCAN 2008, Int. J. Cancer 127 (12) (2010) 2893-2917.

[5] T.P. Kingham, et al., Treatment of cancer in sub-Saharan Africa, Lancet Oncol. 14 (4) (2013) e158-67.

[6] Institute for Health Metrics and Evaluation. The Challenge Ahead: Progress and setbacks in breast and cervical cancer.: Seattle, WA: IHME, 2011. http://www. healthdata.org/sites/default/files/files/policy_report/2011/TheChallengeAhead/ IHME ChallengeAhead FullReport.pdf Accessed: January 2015.

[7] K.S. Louie, S. de Sanjose, P. Mayaud, Epidemiology and prevention of human papillomavirus and cervical cancer in sub-Saharan Africa: a comprehensive review, Trop. Med. Int. Health 14 (10) (2009) 1287-1302.

[8] L.A. Denny, et al., Human papillomavirus, human immunodeficiency virus and immunosuppression, Vaccine 30 (Suppl 5) (2012) F168-F174 (p).
[9] D.K. Singh, et al., Human papillomavirus infection and cervical cytology in HIVinfected and HIV-uninfected Rwandan women, J. Infect. Dis. 199 (12) (2009) 1851-1861.

[10] L. Denny, et al., Human papillomavirus infection and cervical disease in human immunodeficiency virus-1-infected women, Obstet. Gynecol. 111 (6) (2008) 1380-1387.

[11] Ferlay J., et al. GLOBOCAN 2012 v1.0, Cancer Incidence and Mortality Worldwide: IARC CancerBase No. 11 [Internet]. Lyon, France: International Agency for Research on Cancer; 2013. Available from: http://globocan.iarc.fr/, Accessed on 12 January 2015.

[12] UNAIDS, Global Report on the AIDS Epidemic 2013. http://www.unaids.org/en/ media/unaids/contentassets/documents/epidemiology/2013/gr2013/UNAIDS Global_Report_2013_en.pdf Accessed: January 2014.

[13] K.S. Pfaendler, et al., Management of cryotherapy-ineligible women in a screenand-treat cervical cancer prevention program targeting HIV-infected women in Zambia: lessons from the field, Gynecol. Oncol. 110 (3) (2008) 402-407.

[14] G.P. Parham, M.M. Sahasrabuddhe, V.V. Westfall, A.O. King, K.E. Chibwesha, C. Pfaendler, K.S. Mkumba, G. Mudenda, V. Kapambwe, S. Vermund, S.H. Hicks, M.L. Stringer, J.S.A. Chi BH, Implementation of cervical cancer prevention services for HIV-infected women in Zambia: measuring program effectiveness, HIV Therapy 4 (6) (2010) 713-722.

[15] M.H. Mwanahamuntu, et al., Implementation of 'see-and-treat' cervical cancer prevention services linked to HIV care in Zambia, AIDS 23 (6) (2009) N1-5.

[16] M.H. Mwanahamuntu, et al., Integrating cervical cancer prevention in HIV/AIDS treatment and care programmes, Bull. World Health Organ. 86 (8) (2008) D-E.

[17] World Health Organization, World Health Statistics 2014. http://apps.who.int/iris/ bitstream/10665/112738/1/9789240692671_eng.pdf?ua = 1 Accessed: March 2015. 